\title{
Comunicación institucional de crisis y redes sociales en la Covid-19: los casos de Yunquera y Gaucín
}

\author{
Institutional crisis communication and social networks in the Covid-19: the \\ cases of Yunquera and Gaucín
}

\author{
Dra. Andrea Castro-Martínez \\ Universidad de Málaga| Calle de León Tolstoi, s/n, 29010, Málaga | España | \\ https://orcid.org/0000-0002-2775-625X | andreacastro@uma.es

\section{Ignacio Morán-Urdiales} \\ EADE Universidad-University of Wales Trinity Saint David y Universidad de Málaga| Calle \\ Fernández Shaw, 88, 29018, Málaga | España | https://orcid.org/0000-0003-1518-8651| \\ nachourdiales@uma.es

\section{Dr. Pablo Díaz-Morilla} \\ EADE Universidad-University of Wales Trinity Saint David y Universidad de Málaga| Calle \\ Fernández Shaw, 88, 29018, Málaga | España | https://orcid.org/0000-0002-7914- \\ 3391 |pablodmorilla@uma.es
}

Fechas | Recepción: 15/01/2021 | Aceptación: 26/02/2021

Resumen

Los canales digitales y redes sociales constituyen herramientas al servicio de la comunicación institucional tanto para establecer relaciones con los públicos como para informar y legitimar las políticas implementadas. Su uso se ha extendido al ámbito local y es habitual que incluso los municipios pequeños dispongan de perfiles sociales, que han sido una herramienta clave durante la crisis de la Covid-19 para trasladar mensajes a la población.

Esta investigación consiste en un caso de estudio centrado en el uso institucional de Facebook en los municipios malagueños de Yunquera y Gaucín, ambos con menos de 3000 habitantes, y donde esta red ha destacado por su eficacia. Mediante una metodología mixta se combinan entrevistas en profundidad a los

\section{Abstract}

Digital channels and social networks are tools at the service of institutional communication both to establish relations with the public and to inform and legitimise the policies implemented. Their use has spread to the local sphere and it is common for even small municipalities to have social profiles, which have been a key tool during the Covid-19 crisis to convey messages to the population.

This research consists of a case study focusing on the institutional use of Facebook in the Malaga municipalities of Yunquera and Gaucín, both of which have fewer than 3,000 inhabitants, and where this social network has stood out for its effectiveness. Using a mixed methodology, it combines in-depth interviews with councillors, content analysis of social profiles and monitorina of publications durina 
regidores, análisis de contenido de los perfiles sociales y monitorización de las publicaciones durante la pandemia. Facebook ha facilitado enormemente la difusión de información relevante para los vecinos y se ha convertido en el canal digital prioritario para ambos consistorios. También se han incorporado nuevos contenidos como los bandos municipales. La traslación de la comunicación de proximidad propia de estos municipios a las redes sociales y el uso combinado con los perfiles personales de los alcaldes, que han sido preponderantes, ha resultado fundamental en el éxito de sus comunicaciones digitales durante la pandemia. Se incluyen recomendaciones para la gestión de la comunicación digital durante la nueva normalidad en ayuntamientos.

Palabras clave: comunicación institucional, comunicación de crisis, redes sociales, Covid19 , ayuntamientos. the pandemic. Facebook has greatly facilitated the dissemination of relevant information for the residents and has become the priority digital channel for both councils. New content has also been incorporated, such as the municipal sides. The transfer of these municipalities' own communications to social networks and their combined use with the personal profiles of mayors, which have been predominant, has been fundamental in the success of their digital communications during the pandemic. Recommendations are included for the management of digital communication during the new normality in town halls.

\begin{abstract}
Keywords: institutional communication, crisis communication, social networks, Covid-19, town hall.
\end{abstract}

\section{INTRODUCCIÓN}

Internet y las redes sociales han modificado la comunicación entre personas y organizaciones y este nuevo paradigma relacional ha influido también en las instituciones. En este contexto, las páginas web (Almansa y Castillo, 2014), las salas de prensa online (Herrero-Gutiérrez, Martínez-Vallvey, Tapia-Frade, Rey-García, y Cabezuelo-Lorenzo, 2017) o las redes sociales (Padilla, 2016; Kimmons, Veletsianos y Woodward, 2017) han experimentado un gran desarrollo. Las tecnologías de la información y comunicación (TIC) han permitido a las instituciones, incluidas las corporaciones locales (Herrero y Ruano, 2019), y a los actores políticos ganar rapidez y comodidad y "pueden ayudar a las organizaciones a proporcionar información más detallada y útil" (Faber, Budding y Gradus, 2020, p. 1). En este sentido "la comunicación política de los municipios resulta clave para que estos se hagan visibles en la aldea global, promocionando sus actividades y decisiones de gestión" (Mesa-Medina y MarfilCarmona, 2018, p. 18).

La digitalización se ha acelerado por la crisis de la Covid-19, pues la gestión eficaz de la comunicación es de vital importancia para manejar el riesgo (De Sa, Mounier-Jack y Coker, 2009) y resulta "esencial para el control de los brotes" (World Health Organization, 2005, p.1). Durante la pandemia se ha producido una situación de Infodemia (Allahverdipour, 2020) con información poco fiable en rápida evolución, proliferando bulos y noticias falsas (Salaverría et al., 2020; Pérez-Dasilva, Meso-Ayerdi y Mendiguren-Galdospín, 2020) e incrementándose el consumo de redes sociales (Kantar, 2020). La comunicación institucional ha sido imprescindible para que la población comprenda la situación y se muestre colaborativa ante las medidas y restricciones (Costa-Sánchez y López-García, 2020), ya que las relaciones de confianza con los públicos han adquirido aún mayor importancia que antes de la pandemia (Xifra, 2020). 
Este trabajo se centra en Yunquera y Gaucín, dos pueblos enclavados en la malagueña Serranía de Ronda cuya suma de habitantes no alcanza los 4.500 vecinos: 2.869 habitantes en Yunquera y 1.589 en Gaucín (Instituto Nacional de Estadística, 2020). Su elección como objeto de estudio convergen tres factores: en primer lugar, su nivel población, que posibilita que la comunicación institucional sea directa entre gobernantes y gobernados; en segundo lugar, que durante la crisis de la Covid-19 el uso de las redes sociales sea la principal herramienta en la articulación de esa comunicación institucional, ya que sus ayuntamientos han potenciado el uso de Facebook para comunicarse de manera rápida y efectiva con sus habitantes, pese a tratarse de municipios rurales y con una población envejecida que no coincide con el perfil más habitual de usuarios digitales; y en tercer lugar, que fruto de esa rutina comunicativa el alcalde o alcaldesa se implique también a través de sus redes sociales personales.

Esta investigación pone el foco en la comunicación institucional desarrollada por municipios de pequeño tamaño, que no suelen disponer de recursos amplios y emplean una estrategia digital limitada, gestionada habitualmente desde la propia alcaldía. Pretende contribuir a la literatura y visibilizar estrategias de comunicación de crisis que han resultado exitosas al adaptarse a las necesidades de localidades pequeñas y con una población rural en un momento de emergencia sanitaria donde la limitación de la movilidad y la imposición de distanciamiento social impedían su habitual comunicación interpersonal.

El objetivo general de este trabajo es analizar la estrategia de comunicación institucional que siguen los municipios de Yunquera y Gaucín en la esfera digital en el contexto de la crisis de la Covid-19. Como objetivos secundarios se plantean:

- Estudiar el uso que hacen de las redes sociales desde las cuentas municipales.

- Determinar si las cuentas personales de los alcaldes contribuyen a difundir los mensajes de sus consistorios.

- Describir el tipo de contenidos y mensajes que estos municipios emiten en plataformas digitales.

\section{REVISIÓN TEÓRICA}

La comunicación institucional pretende establecer una "relación constante, abierta e interactiva con los ciudadanos, basada en la confianza y credibilidad (Gutiérrez-García y La Porte, 2013, p. 14), por lo que debe emplear todos los medios a su alcance para lograr mensajes eficaces que contribuyan a crear valor añadido (Rodrich, 2012). Las TIC pueden contribuir "a la mejora de los canales de información, comunicación, deliberación y participación de los ciudadanos en la toma de decisiones públicas, haciéndolos más inmediatos, sencillos y efectivos" (Colomo, 2006, p. 28). Uno de sus máximos exponentes en la actualidad son las redes sociales, que se emplean no sólo como herramientas de comunicación política (Rúas y Casero-Ripollés, 2018), sino también como canales fundamentales en el denominado gobierno abierto o gobierno 2. (Lee y Kwak, 2012) --sustentado en la transparencia, la participación y la colaboración (Rodríguez, 2017)- y en la diplomacia digital (Castro-Martínez, 2019). Esto se deriva de que "la conexión entre los medios de comunicación online, y en particular los sociales, y la política se ha convertido en uno de los aspectos centrales de las sociedades contemporáneas" (Krzyżanowski y Tucker, 2018, p. 141). 
Dado el importante valor social de la información de proximidad (López, 2000), internet ofrece a los ayuntamientos una vía eficaz para fomentar la participación e implicación de la ciudadanía (Catalina y García, 2013; Boulianne, 2015) ya que las nuevas tecnologías han modificado el modo en que la población consume información y debate los asuntos públicos (Gil de Zúñiga y Liu, 2017).

Las administraciones locales han desarrollado progresivamente estructuras y canales de comunicación que constituyen direcciones de comunicación para vehicular la comunicación pública de proximidad con los ciudadanos (Vázquez y Fernández, 2014), aunque "la presencia o no de gabinetes de comunicación en ayuntamientos está motivada, en gran medida, por el tamaño y los recursos del mismo. Sin embargo, en muchas ocasiones depende en gran medida de la voluntad de quién gobierne la corporación" (Almansa, 2006, p. 228). Los profesionales de las relaciones públicas tienen aún un importante campo de trabajo en las administraciones locales en cuanto a favorecer la interacción con los stakeholders y promover la participación y la transparencia (Paricio-Esteban, Bruno-Carlos, Alonso-Romero y García-Alcober, 2020) ya que resulta necesaria la profesionalización de los gabinetes de prensa municipales (Cárdenas, 1998). Así, los consistorios organizan su gestión de comunicación conforme a sus necesidades, recursos y rutinas (Gil-García y Pardo, 2005; Campillo, 2011). Las páginas web se han generalizado también, aunque con un importante margen de mejora, en municipios pequeños como herramienta para la rendición de cuentas y la transparencia (Laswad, Fisher y Oyelere, 2005; Fernández-Falero, Trabadela-Robles, Garcés-Botacio y Ruano-López, 2017).

En las últimas décadas en España se han reducido la brecha digital y la desigualdad digital, aunque aún persisten dificultades de acceso y de uso con respecto a las nuevas tecnologías que afectan principalmente a los más desfavorecidos (Torres, 2017). Resulta destacable la generalización en el uso de Internet y la flexibilidad en cuanto a los modos de conexión derivados de la expansión de los teléfonos móviles inteligentes (Calderón, 2019) pero se da una segunda generación de brecha digital ya que hay sujetos que, teniendo acceso a las tecnologías, deciden no usarlas, bien por desconocimiento o por desafección (Cabero y RuizPalmero, 2018).

Por otra parte, la web 2.0 constituye un entorno para el ejercicio de la ciudadanía y se vincula a factores sociales de inclusión digital, como explican Rebollo y Vico (2014) en su estudio sobre el uso de redes sociales por parte de las mujeres rurales andaluzas.

El uso de las redes sociales basado en la información es necesario para mejorar la calidad de vida de los ciudadanos y su uso efectivo de los servicios públicos. Los municipios, que explotan su «proximidad», tienen un papel fundamental como "centro de información», a través de la integración y reconexión de los contenidos de información, ya sean de producción propia o producidos por otras partes interesadas. (Solito y Materassi, 2020, p. 151)

Los consistorios las emplean para comunicarse con los ciudadanos, además de para la promoción turística (Martínez-Valerio, 2012) y visibilizar la marca-ciudad (Molina, Fernández, Gómez y Aranda, 2017). En los municipios españoles de más de 50.000 habitantes "la estrategia de difusión de las tecnologías sociales en los ayuntamientos se ha caracterizado hasta el momento por una apuesta por los departamentos de comunicación" (Criado y Rojas, 2015 , p. 36) y su uso de Twitter, empleado con un tono positivo en la conversación, es 
destacado (Criado y Villodre, 2018). También Facebook ha alcanzado una presencia importante en algunos ayuntamientos (Martínez-Rolán, Piñeiro-Otero y Baamonde-Silva, 2016). De este modo, los consistorios, independientemente de su tamaño, establecen relaciones con sus públicos al tiempo que les trasladan y legitiman sus decisiones (Campillo, 2012).

Los últimos desarrollos tecnológicos (en especial la incorporación de las redes sociales digitales) han supuesto una disminución de la brecha digital entre ayuntamientos según tamaño poblacional, y una mayor autonomía por parte de los municipios más pequeños en el desarrollo de la comunicación política electrónica respecto a niveles superiores de gobierno (provincial, autonómico y estatal). (Català y Penalva, 2020, p. 40)

No obstante, en algunos casos, especialmente en municipios de gran tamaño, el uso que los políticos hacen de las redes sociales no se debe tanto a una estrategia de desintermediación y de contacto directo con los ciudadanos como a que los periodistas y medios de comunicación son uno de los grupos de interés sobre los que impactan sus mensajes en línea (Bernhard y Dohle, 2015). En ocasiones incluso puede darse una correlación negativa entre el uso de los medios sociales como herramienta en la política local y el sentido de comunidad (Effing, van Hillegersberg y Huibers, 2013), aunque la conversación política a través de los medios sociales se asocia positivamente con la participación política (Hyun y Kim, 2015).

En la primera etapa de adopción de redes sociales por parte de las administraciones públicas la urgencia consistió en tener presencia en las distintas plataformas, pese a que en ocasiones se carecía de conocimientos para integrarlas en la estrategia global de comunicación. En la actual etapa de consolidación se han incorporado recursos, se ha profesionalizado su uso y se monitorizan sus resultados para optimizar las comunicaciones (Ure, 2016) e incluso, en los casos más avanzados, se cuenta con guías y estrategias de publicación (Mergel y Bretschneider, 2013). La principal crítica a las redes consiste en "la ausencia de diálogo público y de interacción entre los políticos y la ciudadanía" (Carratalá y Galán, 2016, p. 130) por lo que en una etapa de mayor madurez será necesario "promover modelos más complejos, que atiendan factores mucho más diversos que afectan a las organizaciones públicas" (Criado y Villodre, 2018, p. 621).

En cuanto a las crisis, pese a que cada una tiene particularidades (Castillo-Esparcia, 2010) la comunicación en estas situaciones (Micaletto, 2018) se basa en mantener la credibilidad y confianza a través de una postura proactiva que evite las mentiras y el silencio, así como la improvisación, y a ser posible que emplee a un portavoz como catalizador de las intervenciones (Barquero y Barquero, 2005; Luecke, 2005; Almada, 2009; Xifra, 2009; Rodríguez, Marauri, Armentia y Marín, 2020).

En la actual pandemia se ha producido una situación de infodemia donde han proliferado los bulos, las fake news y las informaciones inexactas (Allahverdipour, 2020; Orso et al., 2020; Apuke y Omar, 2021). Sin embargo, según diversos estudios (Chan et al., 2020; Yu et al., 2020), los medios sociales aplicados de forma adecuada constituyen vías rápidas y eficaces para difundir información clave en esta situación de crisis sanitaria y las instituciones, que en cualquier crisis deben informar de los riesgos evitando el alarmismo (Rodríguez, 2011) para contribuir a que la población mantenga la calma (March, 2011), han jugado un papel fundamental en la gestión de la situación. Desde la irrupción de la COVID-19 la comunicación por parte de los gobiernos locales ha sido clave (Gao y Yu, 2020; Gupta et al., 2020) y muchos 
ayuntamientos han implementado diversas herramientas de comunicación digital, como canales de información mediante servicios de mensajería instantánea como Telegram y WhatsApp (López-Tárraga, 2020; Casero-Ripollés, 2020). Por su parte, la comunicación de los gobiernos locales que no han afrontado de manera coordinada su estrategia en medios sociales durante esta crisis se ha visto resentida (Zeemering, 2021).

\section{METOdOLOGÍA}

Este trabajo se enmarca en los estudios de comunicación institucional (Lammers y Barbour, 2006; Giacomini, Sancino y Simonetto, 2018) y, en concreto, en el uso de redes sociales como estrategia comunicativa (Stier, Bleier, Lietz y Strohmaier, 2018; Olabe y Márquez, 2019). El planteamiento metodológico consiste en un estudio de caso múltiple (Wimmer y Dominick, 1996), ya que permite identificar las peculiaridades del fenómeno analizado. Se ha basado en un enfoque mixto (Teddlie y Tashakkori, 2010) que combina aspectos cualitativos y cuantitativos con el objetivo de alcanzar una perspectiva lo más amplia y completa posible (Berganza y Ruiz San Román, 2005), ya que son técnicas que se complementan (Hernández, Fernández y Baptista, 2010). Como herramientas para la obtención de información se han empleado:

- La revisión bibliográfica, para construir el marco teórico y establecer las prácticas habituales de uso de redes sociales en el marco de la comunicación institucional y las funciones que han desarrollado en el contexto de la pandemia en las estrategias comunicativas de las administraciones locales.

- Las entrevistas en profundidad (Meneses y Rodríguez, 2011) a los máximos responsables de las corporaciones municipales, los regidores de Yunquera y de Gaucín, José Antonio Víquez y Pedro Godino respectivamente.

- El análisis de métricas de las cuentas de Facebook de los ayuntamientos estudiados. Se han excluido los perfiles no relacionados con la gestión informativa de la crisis de la Covid-19 al trascender el objeto de estudio. Si bien ambos municipios cuentan con perfiles en Instagram, se enfocan en la promoción cultural, turística y de ocio. Yunquera también emplea Flickr como repositorio fotográfico y YouTube de forma testimonial -en diez años ha alcanzado únicamente 33.958 visualizaciones- principalmente con grabaciones de los plenos municipales.

- La monitorización de los perfiles personales de los regidores en Facebook, la red social donde tienen presencia pública.

De este modo se ha estudiado el uso que han hecho los consistorios y los alcaldes de las redes sociales en el periodo de análisis, de modo que se han podido identificar los tipos de contenido más empleados, así como las características comunicativas de los mensajes emitidos. El arco temporal de la investigación se circunscribe a la segunda ola de la pandemia. Se obvia la primera ola de la crisis y las semanas de confinamiento estricto porque en estos municipios no tuvo gran efecto y su dimensión a nivel de comunicación institucional fue menor. Este periodo de análisis, entre el 11 de octubre de 2020 y el 11 de enero de 2021, está justificado ante la especial incidencia que la Covid-19 ha tenido en la Serranía de Ronda durante el otoño de 2020. La fecha límite, el 11 de enero, marca el fin de la trayectoria como alcalde de Yunquera de José Antonio Víquez, al haber sido nombrado el 12 de enero de 2021 delegado de Desarrollo Sostenible de la Junta de Andalucía en Málaga. 
Para profundizar en el uso de Facebook y en el impacto que tiene en la comunicación institucional en ambos municipios se ha procedido a analizar, por un lado, los perfiles institucionales de los ayuntamientos, para su posterior comparación con los perfiles personales de sus alcaldes. Se ha abordado de modo cualitativo a través del análisis de contenido de las publicaciones (tabla 1) y con métricas generales del periodo completo con la pretensión de caracterizar los mensajes.

Tabla 1

Plantilla para analizar las cuentas y publicaciones en Facebook

\begin{tabular}{|c|c|c|}
\hline \multirow[t]{3}{*}{ Perfil/ Página } & \multicolumn{2}{|l|}{ Amigos/Fans/Seguidores } \\
\hline & \multicolumn{2}{|c|}{ Información de perfil /Imágenes de perfil } \\
\hline & \multicolumn{2}{|l|}{ Call to action } \\
\hline \multirow[t]{16}{*}{ Publicaciones } & \multirow[t]{3}{*}{ Tipo de publicaciones } & Personales/Institucionales \\
\hline & & Información \\
\hline & & Opinión \\
\hline & \multirow[t]{4}{*}{ Tema } & Sanitario \\
\hline & & Social \\
\hline & & Ocio/ Cultura /Turismo \\
\hline & & Otro \\
\hline & Autoría & Contenido propio/ Ajeno \\
\hline & \multirow[t]{3}{*}{ Contenido audiovisual } & Vídeos \\
\hline & & Fotos \\
\hline & & Gif \\
\hline & \multirow[t]{3}{*}{ Recursos comunicativos } & Links \\
\hline & & Hashtags \\
\hline & & Emoticonos \\
\hline & \multirow[t]{2}{*}{ Lenguaje empleado } & Culto/Técnico /Científico \\
\hline & & Coloquial \\
\hline
\end{tabular}

Fuente: elaboración propia.

Para comprobar las diferencias entre el uso de las páginas y los perfiles antes de que estallara la crisis sanitaria y durante la pandemia, se ha realizado un análisis cuantitativo de los perfiles de las instituciones y los alcaldes en Facebook, en el que se ha comparado su actividad durante febrero de 2020, mes previo a la pandemia, y noviembre del mismo año, en el que los municipios se vieron afectados por la segunda ola. Los datos se han complementado con el estudio cuantitativo pormenorizado de las publicaciones más destacadas y de los hitos 
relevantes en estos municipios a lo largo de la crisis. Las métricas de los perfiles se han extraído de herramientas de monitorización: Facebook Insights y Fan Page Karma. Los datos no aportados por estos programas informáticos se han recolectado de forma manual directamente de los distintos perfiles sociales estudiados.

\section{RESULTADOS}

Facebook es la principal herramienta que tanto el alcalde de Yunquera -José Antonio Víquezcomo el de Gaucín -Pedro Godino- utilizan para comunicarse con los vecinos; a pesar de que las comunicaciones oficiales se siguen albergando en la web municipal de ambos pueblos. Los alcaldes han afrontado la crisis como muchos otros avatares del día a día municipal: "En primera persona" (Víquez, comunicación personal, 8 de enero de 2021). Ambos regidores han articulado su comunicación institucional en un momento en el que los vecinos demandaban mucha más información. "Cuando empezaron a darse los primeros contagios, vimos importante que se trasladara información veraz, así evitábamos todo tipo de conjeturas y conversaciones de pasillo" (Godino, comunicación personal, 9 de enero de 2021).

Esa implicación de carácter personal es uno de los factores en común en ambos municipios en cuanto a la gestión de la comunicación institucional, ya que en los dos casos esta cuestión se arbitra directamente desde la alcaldía. Víquez se encarga personalmente de redactar los bandos, notas y comunicados, ayudado exclusivamente por el informático municipal en cuestiones de formato. En Gaucín, el alcalde es también el responsable de comunicación, aunque en este caso esa labor se lleva a cabo entre varias personas y con ayuda también del Centro Guadalinfo de la zona (Víquez y Godino, comunicación personal, 8 y 9 de enero de 2021).

En cuanto a las rutinas en la gestión de la comunicación previas a la pandemia, en Yunquera el ritmo de difusión "varía mucho, depende de la semana. Hay veces que durante cuatro o cinco días seguidos anunciamos cosas y después estamos una semana sin informar" (Víquez, comunicación personal, 8 de enero de 2021). En el caso de Gaucín, la mañana del alcalde empieza con un repaso a la prensa local y a las redes sociales por si hay algún asunto "sobre el que informar o emitir alguna comunicación oficial" (Godino, comunicación personal, 9 de enero de 2021). Ambos coinciden en que la crisis ha cambiado por completo los ritmos informativos, en Yunquera, y la forma de comunicar, en Gaucín.

En el primer municipio, el uso de las redes sociales para informar a los vecinos sobre cuestiones relevantes se había impuesto hace años. "Me di cuenta de que por más que publicáramos contenido en la web municipal, cuando realmente se enteraban los vecinos era a través de Facebook". Con una particularidad: "Si de verdad era una cuestión muy importante, me veía obligado a utilizar mi perfil personal de Facebook", precisa el alcalde de Yunquera, hasta el punto de que hay vecinos que le han llegado a reprochar que no se han enterado de algún asunto oficial porque no había aparecido en su Facebook personal; a pesar de estar publicada la información en la web del Ayuntamiento y en las redes sociales institucionales (Víquez, comunicación personal, 8 de enero de 2021). Este método de publicación en redes, antes puntual, ahora se ha generalizado "me he visto obligado a convertir mi perfil personal en el altavoz municipal, con comunicaciones diarias", ya que en su cuenta personal siempre se genera mayor interacción con los vecinos que a través de los perfiles institucionales (Víquez, comunicación personal, 8 de enero de 2021). 
El caso de Gaucín deriva en la misma situación, pero el punto de partida es diferente: "El confinamiento nos hizo ver que no teníamos buena comunicación con los vecinos, así que aprovechamos el parón para mejorar la sede electrónica del Ayuntamiento y el uso de las redes sociales" (Godino, comunicación personal, 9 de enero de 2021). En este caso, el alcalde también utiliza su perfil personal en Facebook para trasladar comunicaciones, aunque si éstas son de carácter más oficial, se limita a replicar lo ya publicado en la web municipal y en las redes sociales institucionales.

Ha habido días en los que he tenido que estar constantemente publicando cosas en mi perfil personal, como el día en el que los vecinos de Yunquera fuimos convocados por la Junta de Andalucía para someternos a un cribado. Así avisamos a todos, con mi Facebook y con el sistema de megafonía local, que se usaba antiguamente y que nos ha venido muy bien para una ocasión tan especial. (Víquez, comunicación personal, 8 de enero de 2021)

Y es que el uso de los perfiles personales en redes sociales ha sido de gran importancia: "Tratándose de un municipio pequeño en el que nos conocemos todos, lo que solemos hacer es usar nuestras cuentas personales como altavoz, como amplificador de las comunicaciones que se hacen en perfiles oficiales" (Godino, comunicación personal, 9 de enero de 2021). En cuanto al formato elegido para esas comunicaciones, el alcalde de Yunquera distingue tres niveles:

Los bandos y comunicados oficiales se suben primero a la web; después se replica en redes sociales, donde se comunican y se comentan las novedades de forma más distendida. Por último, si tenemos algo que comunicar con soporte gráfico, también usamos Instagram. (Víquez, comunicación personal, 8 de enero de 2021)

Por su parte, el alcalde de Gaucín precisa que las comunicaciones más institucionales se reservan para Facebook, mientras que la cuenta de Instagram está asociada a la promoción turística, cultural y de ocio del municipio (Víquez y Godino, comunicación personal, 8 y 9 de enero de 2021).

Uno de los formatos utilizados en Facebook por ambos alcaldes durante los últimos meses ha sido el del bando municipal, a pesar de que el tono y la estructura de este tipo de documentos es netamente formal y su contenido en muchos casos es de carácter administrativo. Víquez considera que los bandos han cobrado especial relevancia "porque contenían asuntos relacionados con la pandemia, como restricciones y medidas sanitarias extraordinarias" (Víquez, comunicación personal, 8 de enero de 2021). Tal ha sido el interés por este tipo de documento oficial que el alcalde de Gaucín ha decidido enviarlos por WhatsApp: "Todos los vecinos querían estar al tanto" (Godino, comunicación personal, 9 de enero de 2021).

Respecto a los resultados obtenidos a raíz de estos cambios, ambos coinciden en que el uso de las redes sociales y su implicación personal ha sido clave. "Los vecinos están mucho más enterados", asegura Víquez, mientras que Godino celebra que los resultados en redes sociales "son cada vez mejores, con un incremento de visitas e interacciones". Preguntados por si prevén mantener estas rutinas en cuanto a la gestión de la comunicación institucional, ambos coinciden en que sí; y en el caso de Gaucín ya se está trabajando en un canal de WhatsApp municipal que llegue a todos los vecinos. No obstante, ninguno monitoriza las publicaciones, ya que no conocen ni utilizan la medición en Facebook, aunque se muestran convencidos de 
que a raíz de la pandemia y, sobre todo de la segunda ola, las interacciones y el impacto de sus publicaciones aumentaron.

Ambos alcaldes advierten de la importancia de luchar contra la desinformación - usan los términos "bulo" y "fake news" - en situaciones comprometidas como las ocasionadas por la pandemia. Como solución, apuestan por una comunicación institucional "más frecuente" (Víquez, comunicación personal, 8 de enero de 2021) y "muy cercana, que sea de andar por casa" (Godino, comunicación personal, 9 de enero de 2021).

Resulta relevante el alcance de las publicaciones teniendo en cuenta que tanto en las fanpages como las cuentas personales aglutinan comunidades superiores a la población de los municipios (tabla 1).

Tabla 2

Comparativa entre páginas municipales y perfiles personales de los regidores

\begin{tabular}{|l|c|c|c|c|}
\hline \multicolumn{1}{|c|}{ Municipio } & Página/Perfil & Fans/Amigos & $\begin{array}{c}\text { Media de } \\
\text { publicaciones diarias }\end{array}$ & Tasa de interacción \\
\hline Yunquera & Ayuntamiento & 4.300 & 1.1 & $0.79 \%$ \\
\hline Yunquera & Alcalde & 4.248 & $\mathrm{xx}$ & - \\
\hline Gaucín & Ayuntamiento & 2.300 & 0.2 & $0.24 \%$ \\
\hline Gaucín & Alcalde & 1.987 & 0.35 & - \\
\hline
\end{tabular}

Fuente: elaboración propia. Facebook y Fan Page Karma.

La participación es muy superior en los perfiles personales a la de las páginas oficiales, donde apenas se registran interacciones (tablas 2 y 3). Es una constante en todo el periodo de análisis el mayor atractivo de los perfiles personales de los alcaldes y queda patente también en los acontecimientos más relevantes durante la crisis (tabla 4).

El impacto y las interacciones principales se producen siempre en torno a cuestiones relacionadas con el día a día del municipio, aunque de temática variada. En las publicaciones en los perfiles institucionales no aparece en lugar destacado las informaciones o comunicaciones relacionadas con la pandemia, asunto que sí ocupa algunas de las publicaciones con más interacciones, pero en los perfiles personales de los alcaldes.

Tabla 3

Publicaciones más relevantes de Yunquera

\begin{tabular}{|l|l|l|l|l|}
\hline Fuente & Publicación & $\begin{array}{l}\text { Me gusta/Me } \\
\text { encanta/Me } \\
\text { sorprende }\end{array}$ & Comentarios & $\begin{array}{l}\text { Veces } \\
\text { compartido }\end{array}$ \\
\hline Ayto. & $\begin{array}{l}\text { Cartel sobre la Sierra de las Nieves y su } \\
\text { próxima declaración como Parque Nacional. } \\
(5 / 12 / 2020)\end{array}$ & 93 & 65 \\
\cline { 2 - 5 } & $\begin{array}{l}\text { Celebración del Día de la Mujer Rural. } \\
(16 / 10 / 2020)\end{array}$ & 89 & 30 & 20 \\
\hline
\end{tabular}




\begin{tabular}{|c|c|c|c|c|}
\hline & $\begin{array}{l}\text { Vídeo sobre las fiestas navideñas. } \\
(2 / 12 / 2020)\end{array}$ & 65 & 22 & 56 \\
\hline & $\begin{array}{l}\text { Video sobre la celebración del Día de la } \\
\text { Mujer Rural. (16/10/2020) }\end{array}$ & 57 & 35 & 23 \\
\hline & $\begin{array}{l}\text { Activación del Plan de Emergencia } \\
\text { Municipal. }(13 / 11 / 2020)\end{array}$ & 47 & 21 & 45 \\
\hline \multirow[t]{5}{*}{ Regidor } & $\begin{array}{l}\text { Texto de enhorabuena a los vecinos por su } \\
\text { buen comportamiento respecto al cribado } \\
\text { masivo. }(2 / 12 / 2020)\end{array}$ & 517 & 210 & 114 \\
\hline & $\begin{array}{l}\text { Reconocimiento gastronómico a un } \\
\text { restaurante de la Sierra de las Nieves. Texto } \\
\text { del alcalde comentando una noticia. } \\
(23 / 10 / 2020)\end{array}$ & 486 & 206 & 58 \\
\hline & $\begin{array}{l}\text { Bienvenida a la primera mujer policía local } \\
\text { en la Sierra de las Nieves. }(21 / 12 / 2020)\end{array}$ & 383 & 86 & 27 \\
\hline & $\begin{array}{l}\text { Publicación con motivo del fallecimiento de } \\
\text { un vecino. }(26 / 11 / 2020)\end{array}$ & 308 & 300 & 53 \\
\hline & $\begin{array}{l}\text { Foto panorámica de Yunquera con la Sierra } \\
\text { de las Nieves nevada acompañada de una } \\
\text { breve alabanza del alcalde. }(11 / 1 / 2021)\end{array}$ & 291 & 23 & 47 \\
\hline
\end{tabular}

Fuente: elaboración propia. Facebook.

En los perfiles institucionales destacan aquellas publicaciones que apelan al sentido de pertenencia a la comunidad o que especifican cuestiones relacionadas con las fiestas navideñas o con la meteorología. En el caso de los perfiles de los alcaldes, los impactos son más variados; y aparte de los hitos producidos por la pandemia, se abordan otras cuestiones de carácter social o promocional.

Tabla 4

Publicaciones más relevantes de Gaucín

\begin{tabular}{|c|c|c|c|c|}
\hline Fuente & Publicación & $\begin{array}{l}\text { Me gusta/ Me } \\
\text { encanta/ Me } \\
\text { sorprende }\end{array}$ & Comentarios & $\begin{array}{l}\text { Veces } \\
\text { compartido }\end{array}$ \\
\hline \multirow[t]{5}{*}{ Ayto. } & $\begin{array}{l}\text { Fotografía sobre la recepción en Gaucín de } \\
\text { los Reyes Magos. }(5 / 1 / 2021)\end{array}$ & 8 & 0 & 5 \\
\hline & $\begin{array}{l}\text { Comienzo de unas obras de remodelación } \\
\text { en una calle del municipio. }(14 / 10 / 2020)\end{array}$ & 5 & 0 & 7 \\
\hline & $\begin{array}{l}\text { Restricciones sanitarias aprobadas por la } \\
\text { Junta de Andalucía. }(30 / 10 / 2020)\end{array}$ & 2 & 0 & 10 \\
\hline & Anuncio de cribado masivo. $(8 / 1 / 2021)$ & 5 & 0 & 4 \\
\hline & $\begin{array}{l}\text { Normas sobre la visita al cementerio con } \\
\text { motivo de la festividad de Todos los Santos. }\end{array}$ & 5 & 0 & 4 \\
\hline
\end{tabular}




\begin{tabular}{|c|c|c|c|c|}
\hline & $(14 / 10 / 2020)$ & & & \\
\hline \multirow[t]{5}{*}{ Regidor } & $\begin{array}{l}\text { Anuncio con fotografías de la adquisición de } \\
\text { equipos de desinfección y purificadores. } \\
(21 / 11 / 2020)\end{array}$ & 219 & 43 & 154 \\
\hline & $\begin{array}{l}\text { Felicitación institucional navideña. Breve } \\
\text { texto acompañado de una postal. } \\
(23 / 12 / 2020)\end{array}$ & 172 & 87 & 8 \\
\hline & $\begin{array}{l}\text { Visita al cementerio con motivo del Día de } \\
\text { Todos Los Santos. Texto y fotografías. } \\
(3 / 11 / 2020)\end{array}$ & 152 & 36 & 13 \\
\hline & $\begin{array}{l}\text { Evolución de la pandemia en el municipio. El } \\
\text { alcalde informa de que hay cuatro vecinos } \\
\text { contagiados. (16/11/2020) }\end{array}$ & 144 & 57 & 82 \\
\hline & $\begin{array}{l}\text { Fotografía del alcalde y texto explicativo } \\
\text { sobre una acción solidaria. }(24 / 12 / 2020)\end{array}$ & 132 & 22 & 12 \\
\hline
\end{tabular}

Fuente: elaboración propia. Facebook.

Los acontecimientos de mayor importancia a lo largo de la segunda ola se abordaron de forma simultánea en las fanpages y en los perfiles personales, donde su repercusión fue mayor (tabla 4). Además, se produce en ambos municipios un hito que requiere de una necesidad comunicativa a nivel institucional: la población va a ser sometida a cribados por parte de la Junta de Andalucía, hecho que generará una especial interacción en los perfiles sociales. Igual sucede con el nombramiento de José Antonio Víquez, que ha modificado al alza las métricas de su perfil en los últimos días del análisis.

Tabla 5

Comparativa entre perfiles institucionales y personales en los hitos comunicativos

\begin{tabular}{|c|c|c|c|c|c|}
\hline Hito & Fecha & $\begin{array}{l}\text { Página/ } \\
\text { Perfil }\end{array}$ & $\begin{array}{l}\text { Me gusta/ } \\
\text { Me encanta }\end{array}$ & Comentarios & $\begin{array}{l}\text { Veces } \\
\text { compartido }\end{array}$ \\
\hline \multirow[t]{2}{*}{ Cribado masivo (Yunquera) } & \multirow[t]{2}{*}{$27 / 11 / 2020$} & Regidor & 131 & 32 & 65 \\
\hline & & Ayto. & 27 & 0 & 7 \\
\hline \multirow[t]{2}{*}{ Cribado masivo (Gaucín) } & \multirow[t]{2}{*}{$8 / 1 / 2021$} & Regidor & 94 & 5 & 4 \\
\hline & & Ayto. & 5 & 0 & 4 \\
\hline \multirow{2}{*}{$\begin{array}{l}\text { Aprobación de los } \\
\text { presupuestos de Yunquera } \\
\text { para } 2021\end{array}$} & \multirow[t]{2}{*}{$30 / 11 / 2021$} & Regidor & 205 & 37 & 16 \\
\hline & & $\begin{array}{l}\text { Ayto. } \\
\text { (Con } \\
\text { enlace } \\
\text { para ver }\end{array}$ & 8 & 0 & 0 \\
\hline
\end{tabular}




\begin{tabular}{|l|l|l|l|l|l|}
\hline & & $\begin{array}{l}\text { la sesión } \\
\text { plenaria) }\end{array}$ & & & \\
\hline $\begin{array}{l}\text { Bando del alcalde de Gaucín } \\
\text { sobre las restricciones } \\
\text { sanitarias }\end{array}$ & $10 / 1 / 2021$ & Regidor & 33 & 0 & 4 \\
\cline { 2 - 6 } & Ayto. & 1 & 0 & 0 \\
\hline
\end{tabular}

Fuente: elaboración propia. Facebook.

En el caso de los perfiles institucionales se utiliza un tono más formal y con algunos vicios más propios de un lenguaje administrativo, como puede ser el empleo habitual de fórmulas como 'se informa de que', 'se hace saber que' o, en el caso de Gaucín, el comienzo de todas las comunicaciones de carácter institucional con un 'Buenas tardes vecinos y amigos' y vayan firmadas por el alcalde. Por el contrario, en el caso de sus perfiles personales, se utiliza un tono más distendido, con expresiones coloquiales -'pequeñajos', 'dejar de mirarnos el ombligo', 'cerrozajo', 'radio-patio'- e incluso con recursos como el humor o la ironía. Este uso distendido se amplía aún más en las interacciones que mantienen con los usuarios que comentan las publicaciones.
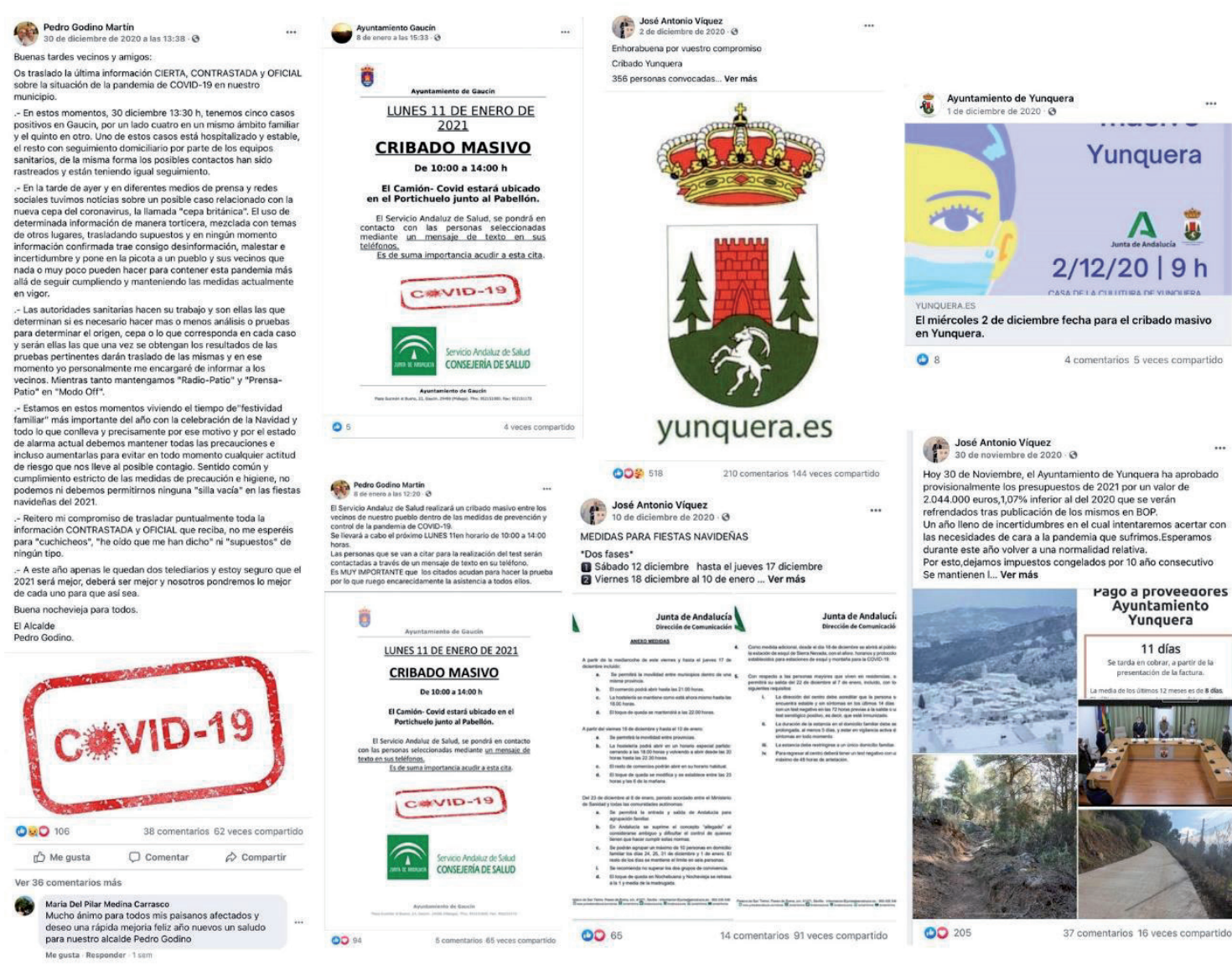

Imagen 1. Publicaciones en Facebook de las cuentas oficiales y los perfiles de los alcaldes.

Fuente: Facebook.

Uno de los formatos que se ha visto afectado por esta dualidad del lenguaje es el de los bandos, un documento de carácter administrativo que ha cobrado relevancia y popularidad a raíz de la pandemia. Tradicionalmente, es un comunicado oficial emitido por el máximo 
responsable político de un ayuntamiento y que contiene información importante sobre el cumplimiento de ordenanzas municipales. La pandemia ha convertido este documento en algo relevante, ya que han incluido, en aplicación de las medidas aprobadas por las administraciones competentes, la reducción de la movilidad y otro tipo de restricciones por motivos sanitarios. La importancia de conseguir que estas medidas fueran conocidas por la población ha llevado a los alcaldes a publicar los bandos en las redes, tanto institucionales como personales; acompañados en algunos casos de comentarios explicativos en un lenguaje más distendido.

El análisis comparativo de las publicaciones realizadas en los perfiles y páginas de Facebook antes de la pandemia y durante la segunda ola muestra que la rutina en redes sociales de los perfiles institucionales y de los alcaldes de Gaucín y Yunquera se vio alterada con la llegada de la crisis. En el caso del perfil institucional del Ayuntamiento de Gaucín, el incremento de publicaciones en noviembre respecto a febrero es de un 33\%; mientras que el nivel de interacciones se sitúa en 24,5 interacciones por publicación en febrero frente a las 2,37 interacciones por publicación en noviembre. Esta bajada en cuanto al nivel de interacciones se debe a que en noviembre el perfil personal del alcalde es el que capitaliza todas las comunicaciones de carácter institucional. En concreto, en el caso del perfil personal del regidor de Gaucín, Pedro Godino, el incremento de publicaciones en noviembre respecto a febrero es de un 200\%; mientras que el nivel de interacciones se sitúa en 61,43 interacciones por publicación en febrero frente a las 88,5 interacciones por publicación de noviembre.

El análisis cuantitativo de las redes sociales de Yunquera arroja un resultado similar, aunque en este caso, tanto el perfil institucional como el personal del alcalde tenían también una actividad intensa en el periodo previo a la pandemia; lo que no evita que el tráfico se intensifique con la llegada de la segunda ola y de un incremento de contagios en este municipio. Específicamente, el perfil institucional del Ayuntamiento de Yunquera registra un 65 por ciento más de publicaciones en noviembre respecto a febrero; mientras que el nivel de interacciones se sitúa en 21,6 interacciones por publicación en febrero, frente a las 17,2 interacciones de media por publicación en noviembre. Al igual que ocurre con Gaucín, las interacciones se centrarán más en el perfil personal del alcalde, que adquiere también un carácter oficial. En concreto, en el caso del perfil personal del alcalde de Yunquera, José Antonio Víquez, el incremento de publicaciones en noviembre respecto a febrero es de un 320\%; mientras que el nivel de interacciones se sitúa en 105,6 interacciones por publicación en febrero frente a las 107,59 interacciones por publicación en noviembre. En este caso, la principal diferencia se sitúa en el número de publicaciones, que se incrementa notablemente.

\section{DISCUSIÓN Y CONCLUSIONES}

Los resultados indican, en la línea de trabajos precedentes (Padilla, 2016; Criado y Villodre, 2018; Olabe y Márquez, 2019), que las redes sociales son un canal de gran utilidad en la comunicación institucional, ya que, coincidiendo entre otros con Solito y Materassi (2020), contribuyen a que la ciudadanía permanezca informada. A través de su uso los consistorios de Yunquera y Gaucín han explicado las políticas adoptadas (Campillo, 2012) y aportando información útil y detallada (Faber et al., 2020) han logrado la colaboración ciudadana (CostaSánchez y López-García, 2020). La relevancia de la información de proximidad, clave en la política local (López, 2000; Vázquez y Fernández, 2014), queda patente durante esta crisis, 
donde los propios alcaldes señalan la necesidad de luchar contra bulos y noticias falsas, coincidiendo con otros estudios (Pérez-Dasilva et al., 2020; Salaverría et al., 2020). Los ayuntamientos analizados, al no disponer de gabinetes de comunicación debido a su tamaño y escasez de recursos (Almansa, 2006), han ideado un esquema de trabajo adaptado a sus necesidades (Campillo, 2011) y aprovechado las ventajas de las redes para vehicular su comunicación de crisis de forma efectiva.

La pandemia ha modificado sus rutinas comunicativas, especialmente en cuanto a canales, contenidos y frecuencia de publicación. Se ha potenciado el uso de Facebook, ampliando el número y la tipología de los mensajes emitidos para incluir, por ejemplo, los bandos municipales. La efectividad se ha logrado combinando las cuentas corporativas con el peso que tienen las figuras de los regidores, que han actuado como amplificadores de los mensajes institucionales a través de sus perfiles personales, convirtiéndose a menudo en las fuentes prioritarias de información para los vecinos. Pese a la falta de profesionalización y a la improvisación inicial propia de una situación inédita, el éxito ha residido en la traslación orgánica y rápida a las redes de la comunicación de proximidad que caracteriza a los municipios de tamaño reducido, que se ha adaptado a las demandas y necesidades de la población. Las redes sociales ligadas a los consistorios se han constituido como fuentes de información fiables y útiles durante esta crisis.

Este trabajo alcanza sus objetivos al dar cuenta de la estrategia de comunicación digital institucional aplicada durante la pandemia en los municipios de Yunquera y Gaucín, así como del uso tanto de las cuentas municipales en redes sociales como de las de sus regidores.

Pese a la efectividad de la estrategia empleada por estos municipios durante la crisis de la Covid-19, se incluyen, con un carácter propositivo para el periodo de 'nueva normalidad', apreciaciones sobre la comunicación online en ayuntamientos de pequeño tamaño:

- Potenciar la marca del consistorio en redes de modo que resulte una fuente de información preferente para los vecinos.

- Emplear las cuentas personales de los cargos públicos como refuerzo de los perfiles oficiales, evitando que resulten canales sustitutivos. Se puede potenciar el tráfico desde los perfiles personales hacia la web y las redes oficiales con llamadas a la acción, retroalimentación y compartiendo sus contenidos en lugar de duplicarlos.

- Establecer una monitorización sistemática de las cuentas que permita conocer los resultados de las publicaciones para optimizar el esfuerzo de gestión digital y potenciar sus resultados.

- Profesionalizar la comunicación institucional a través de la incorporación, en la medida de lo posible, de expertos en estrategia online.

- Mantener el estilo directo y cercano que ha dado buen resultado en la comunicación de proximidad.

Las limitaciones de esta investigación son las propias de un caso de estudio, aunque los resultados pueden ser de utilidad para los profesionales de las administraciones públicas locales. La digitalización y las herramientas de comunicación ligadas a las TIC en municipios constituyen un área interesante para futuras líneas de trabajo, donde se puede profundizar en 
asuntos como la participación, la monitorización de perfiles corporativos y la integración con otras herramientas.

\section{Referencias}

Allahverdipour H. (2020). Global Challenge of Health Communication: Infodemia in the Coronavirus Disease (COVID-19) Pandemic. I Educ Community Health,7(2), 65-67. Recuperado de http://iech.umsha.ac.ir/article-1-1000-en.html

Almada, A. A. (2009). Marketing de crisis. Herramientas concretas para afrontar la actual crisis económica. Madrid: Pirámide.

Almansa, A. (2006). Gabinetes de comunicación. Estudio sobre su presencia en las organizaciones, Anàlisi: quaderns de comunicació i cultura, (34), 223-237. Recuperado de https://www.raco.cat/index.php/Analisi/article/view/55454

Almansa, A. \& Castillo-Esparcia, A. (2014). Comunicación Institucional en España. Estudio del uso que los diputados españoles hacen de las TIC en sus relaciones con la ciudadanía. Chasqui Revista Latinoamericana de Comunicación, 26. Recuperado de https://revistachasqui.org/index. php/chasqui/article/view/250/694

Apuke, O. D., \& Omar, B. (2021). Fake news and COVID-19: modelling the predictors of fake news sharing among social media users. Telematics and Informatics, 56, 101475. https://doi.org/10.1016/j.tele.2020.101475

Barquero, J. D. \& Barquero, M. (2005). Manual de Relaciones Públicas, Comunicación y Publicidad. Barcelona: Editorial Gestión 2000.

Berganza, R. \& Ruiz San Román, J. A. (2005). Investigar en comunicación. Madrid: McGraw Hill.

Bernhard, U. \& Dohle, M. (2015) Local Politics Online: The Influence of Presumed Influence on Local Politicians' Online Communication Activities in Germany, Local Government Studies, 41(5), 755773, https://doi.org/10.1080/03003930.2015.1028624

Boulianne, S. (2015). Social media use and participation: A meta-analysis of current research. Information, communication \& society, 18(5), 524-538. https://doi.org/10.1080/1369118X.2015.1008542

Cabero, J., y Ruiz-Palmero, J. (2018). Las Tecnologías de la información y la comunicación para la inclusión: reformulando la brecha digital, International Journal of Educational Research and Innovation (IJERI), 9, 16-30, http://hdl.handle.net/11441/66918

Calderón Gómez, D. (2019). Una aproximación a la evolución de la brecha digital entre la población joven en España (2006-2015). RES. Revista Española de Sociología, 28(1), 27-44. http://dx.doi.org/10.22325/fes/res.2018.16)

Campillo Alhama, C. (2011). La dirección de comunicación municipal: estructuras, procesos y entidades declarantes, Revista Internacional de Relaciones Públicas, 1(2), 41-60. Recuperado de http://hdl.handle.net/10630/5027

Campillo Alhama, C. (2012). Investigación en comunicación municipal: estudios y aportaciones académicas, Vivat $\quad$ Academia, 1035-1048. doi: http://dx.doi.org/10.15178/va.2011.117E.1035-1048 
Cárdenas Rica, M. L. (1998). Profesionalización de los gabinetes de prensa municipales, Ámbitos Revista Internacional de Comunicación, 1, 131-141. Recuperado de http://hdl.handle.net/11441/67266

Carratalá, A. \& Galán, M. (2016). Estrategias comunicativas en Twitter de los ayuntamientos españoles gobernados por plataformas ciudadanas, Revista F@ro, 2(24), 126-150.

Casero-Ripollés, A. (2020). Servicios móviles de mensajería instantánea y Covid-19: oportunidades y limitaciones para la comunicación institucional. Anuario ThinkEPI, 14, e14d05. https://doi.org/10.3145/thinkepi.2020.e14d05

Castillo-Esparcia, A. (2010). Introducción a las Relaciones Públicas. Málaga: Editorial Instituto de Investigación en Relaciones Públicas.

Castro-Martínez, A. (2019). Ciberdiplomacia y comunicación institucional: La presencia de la diplomacia digital española en redes sociales. Revista Estudios Institucionales, 6(10), 45-72. doi: https://doi.org/10.5944/eeii.vol.6.n.10.2019.23243

Català, LI. \& Penalva, C. (2020). El desarrollo del e-Gobierno en los pequeños municipios de la Comunidad Valenciana (España): más espejo que cristal. AGER: Revista de Estudios sobre Despoblación y Desarrollo Rural (Journal of Depopulation and Rural Development Studies), (29), 39-77. doi: https://doi.org/10.4422/ager.2020.02

Catalina García, B. \& García Jiménez, A. (2013). Herramientas interactivas y participación ciudadana en los ayuntamientos españoles, Ámbitos Revista Internacional de Comunicación, 22, 1-19. doi: http://dx.doi.org/10.12795/Ambitos.2013.i22.15

Chan, A.K.M., Nickson, C.P., Rudolph, J.W., Lee, A. and Joynt, G.M. (2020), Social media for rapid knowledge dissemination: early experience from the COVID-19 pandemic. Anaesthesia, 75, 1579-1582. https://doi.org/10.1111/anae.15057

Colombo, C. (2006). Innovación democrática y TIC, ¿̇ hacia una democracia participativa?. IDP. Revista de Internet, Derecho y Política, (3), 28-40. Recuperado de https://dialnet.unirioja.es/servlet/articulo?codigo=2119691

Costa-Sánchez, C. \& López-García, X. (2020). Comunicación y crisis del coronavirus en España. Primeras lecciones. El profesional de la información, 29(3), e290304. doi: https://doi.org/10.3145/epi.2020.may.04

Criado, J. I. \& Rojas Martin, F. (2015). El impacto de las redes sociales digitales en las administraciones locales. Mitos y realidades en el caso español. IDP. Revista de Internet, Derecho y Política, (20),25-42 Recuperado de https://dialnet.unirioja.es/servlet/articulo?codigo=5582991

Criado, J. I. \& Villodre, J. (2018). Comunicando datos masivos del sector público local en redes sociales. Análisis de sentimiento en Twitter. El profesional de la información, 27(3), 614-623. doi: https://doi.org/10.3145/epi.2018.may.14

De Sa, J., Mounier-Jack, S., \& Coker, R. (2009). Risk communication and management in public health crises, Public health, 123(10), 643-644. doi: https://doi.org/10.1016/j.puhe.2009.07.017

Effing R., van Hillegersberg J., Huibers T.W.C. (2013) Social Media Participation and Local Politics: A Case Study of the Enschede Council in the Netherlands. In: Wimmer M.A., Tambouris E., Macintosh A. (eds) Electronic Participation. ePart 2013. Lecture Notes in Computer Science, vol 8075. Springer. https://doi.org/10.1007/978-3-642-40346-0 6 
Faber, B., Budding, T., \& Gradus, R. (2020). Assessing social media use in Dutch municipalities: Political, institutional, and socio-economic determinants. Government Information Quarterly, 37(3), 101484. doi: https://doi.org/10.1016/j.giq.2020.101484

Fernández-Falero, M. R., Trabadela-Robles, J., Garcés-Botacio, I., \& Ruano-López, S. (2017). Comunicación política de los ayuntamientos a través de sus webs. Caso de Extremadura. El profesional de la información, 26(3), 404-410. doi: https://doi.org/10.3145/epi.2017.may.06

Feezell, J. T. (2018). Agenda Setting through Social Media: The Importance of Incidental News Exposure and Social Filtering in the Digital Era. Political Research Quarterly, 71(2), 482-494. https://doi.org/10.1177/1065912917744895

Gao, X. y Yu, J. (2020) Public governance mechanism in the prevention and control of the COVID-19: information, decision-making and execution, Journal of Chinese Governance, 5(2), 178-197, https://doi.org/10.1080/23812346.2020.1744922

Giacomini, D., Sancino, A. \& Simonetto, A. (2018). The introduction of mandatory inter-municipal cooperation in small municipalities: Preliminary lessons from Italy, International Journal of Public Sector Management, 31(3), 331-346. doi: https://doi.org/10.1108/IJPSM-03-2017-0071

Gil de Zúñiga, H. y \& Liu, J.H. (2017) Second Screening Politics in the Social Media Sphere: Advancing Research on Dual Screen Use in Political Communication with Evidence from 20 Countries, Journal of Broadcasting \& Electronic Media, 61(2), 193-219, https://doi.org/10.1080/08838151.2017.1309420

Gil-García, J. R., \& Pardo, T. A. (2005). E-government success factors: Mapping practical tools to theoretical foundations. Government information quarterly, 22(2), 187-216. https://doi.org/10.1016/i.giq.2005.02.001

Gupta, S., Nguyen, T. D., Rojas, F. L., Raman, S., Lee, B., Bento, A., ... \& Wing, C. (2020). Tracking public and private responses to the COVID-19 epidemic: evidence from state and local government actions (No. w27027). National Bureau of Economic Research. DOI 10.3386/w27027

Gutiérrez-García, E. \& La Porte, T. (2013). Tendencias emergentes en la comunicación de instituciones. Barcelona: UOC Press.

Hernández, R., Fernández, C., \& Baptista P. (2010). Metodología de la Investigación. Distrito Federal de México: McGraw Hill.

Herrero-Gutiérrez, F. J., Martínez-Vallvey, F., Tapia-Frade, A., Rey-García, P., \& Cabezuelo-Lorenzo, F. (2017). Transparencia en el sector público a través de salas de prensa online. Gestión de recursos colectivos y su información en webs municipales. El profesional de la información, 26(3), 421-429. doi: https://doi.org/10.3145/epi.2017.may.08

Herrero Gutiérrez, F. \& Ruano López, S. (2019). Transparencia municipal y participación ciudadana en la democracia española. Estudio de caso: Comunidad de Madrid. Chasqui. Revista Latinoamericana de Comunicación, 4(139), 449-468. doi: https://doi.org/10.16921/chasqui.v0i139.3354

Hyun, K. D., \& Kim, J. (2015). Differential and interactive influences on political participation by different types of news activities and political conversation through social media. Computers in Human Behavior, 45, 328-334. https://doi.org/10.1016/i.chb.2014.12.031.

Instituto Nacional de Estadística (2020). Malaga: población por municipios. INE. Recuperado de https://www.ine.es/jaxiT3/Datos.htm?t=2882\#!tabs-tabla 
Kantar (2020). COVID-19 Barometer: Consumer attitudes, media habits and expectations, Kantar. Recuperado de https://www.kantar.com/Inspiration/Coronavirus/COVID-19-BarometerConsumer-attitudes-media-habits-and-expectations

Kimmons, R., Veletsianos, G. \& Woodward, S. Institutional Uses of Twitter in U.S. Higher Education. Innov High Educ, 42, 97-111 (2017). doi: https://doi.org/10.1007/s10755-016-9375-6

Krzyżanowski, M., \& Tucker, J. A. (2018). Re/constructing politics through social \& online media: Discourses, ideologies, and mediated political practices. Journal of Language and Politics, 17(2), 141-154. https://doi.org/10.1075/jlp.18007.krz

Lammers, J.C \& Barbour, J.B. (2006). An Institutional Theory of Organizational Communication, Communication Theory, 16(3), 356-377. doi: https://doi.org/10.1111/j.14682885.2006.00274.x

Laswad, F., Fisher, R., \& Oyelere, P. (2005). Determinants of voluntary Internet financial reporting by local government authorities. Journal of accounting and public policy, 24(2), 101-121. https://doi.org/10.1016/i.jaccpubpol.2004.12.006

Lee, G., \& Kwak, Y. H. (2012). An open government maturity model for social media-based public engagement. Government information quarterly, 29(4), 492-503. https://doi.org/10.1016/i.giq.2012.06.001

López-Tárraga, A. B. (2020). Comunicación de crisis y Ayuntamientos: el papel de Telegram durante la crisis sanitaria de la COVID-19, RAEIC, Revista de la Asociación Española de Investigación de la Comunicación, 7(14), 104-126. doi: https://doi.org/10.24137/raeic.7.14.5

López García, X. (2000). La comunicación del futuro se escribe con L de local, Ámbitos Revista Internacional de Comunicación, 5, 107-117. Recuperado de http://hdl.handle.net/11441/67340

Luecke, L. (2005). Gestión de crisis convertirlas en oportunidades. Barcelona: Ediciones Deusto.

March Cerdá, J. C. (2011). El riesgo de una mala comunicación de riesgos. Revista de Comunicación y Salud, 1(2), 61-66. doi: http://doi.org/10.35669/revistadecomunicacionysalud.2011.1(2).61-66

Martínez-Rolán, X., Piñeiro-Otero, T., \& Baamonde-Silva, X. M. (2016). El desafío de la transparencia en la comunicación digital. Un estudio de caso sobre las webs municipales de Galicia y Norte de Portugal. Observatorio (OBS*), 10(4), 35-55. Recuperado de http://www.scielo.mec.pt/scielo.php?script=sci_arttext\&pid=S164659542016000500003\&lng=pt\&tlng=es

Martínez-Valerio, L. (2012). Estrategias de promoción turística a través de Facebook. Palabra Clave, 15(2), 318-338. Recuperado de https://www.redalyc.org/articulo.oa?id=649/64923562006

Meneses, J. \& Rodríguez, D. (2011). El cuestionario y la entrevista. Barcelona: UOC.

Mergel, I. \& Bretschneider, S. I. (2013). A three-stage adoption process for social media use in government. Public Administration Review, 3, 390-400. doi: https://doi.org/10.1111/puar.12021

Mesa-Medina, Ó. \& Marfil-Carmona, R. (2018). Diseño y propuesta de un modelo de análisis para la gestión de la comunicación corporativa local. Ámbitos. Revista Internacional de Comunicación, 3(42), 18-39._doi: http://dx.doi.org/10.12795/Ambitos.2018.i42.02 
Micaletto Belda, J. (2018). Principios fundamentales de la comunicación en crisis: una configuración táctica. Razón Y Palabra, 22(2_101), 503-517. Recuperado de http://www.revistarazonypalabra.org/index.php/ryp/article/view/1079

Molina, A., Fernández, A. C., Gómez, M., \& Aranda, E. (2017). Differences in the city branding of European capitals based on online vs. offline sources of information. Tourism Management, 58, 28-39. doi: https://doi.org/10.1016/j.tourman.2016.10.005

Olabe, F. M. \& Márquez López. J. A. (2019). Integración de Twitter y Facebook en la comunicación de la administración local: el Ayuntamiento de Elche como caso de estudio, Miguel Hernández Communication Journal, 10(1), 57-81. doi: http://dx.doi.org/10.21134/mhcj.v10i0.275

Orso, D., Federici, N., Copetti, R., Vetrugno, L., \& Bove, T. (2020). Infodemic and the spread of fake news in the COVID-19-era. European journal of emergency medicine : official journal of the European Society for Emergency Medicine, 27(5), 327-328. https://doi.org/10.1097/MEJ.0000000000000713

Padilla Castillo, G. (2016). Redes sociales institucionales de éxito: los casos de @policia y @guardiacivil en España. Opción: Revista de Ciencias Humanas y Sociales, (12), 85-109. Recuperado de https://dialnet.unirioja.es/descarga/articulo/5852298.pdf

Paricio-Esteban, P., Bruno-Carlos, T., Alonso-Romero, E., \& García-Alcober, M. P. (2020). Webs y portales de transparencia para la participación ciudadana en la gestión de las relaciones públicas con los stakeholders locales. Profesional de la información, 29(3), e290335. doi: https://doi.org/10.3145/epi.2020.may.35

Pérez-Dasilva, J. A., Meso-Ayerdi, K. \& Mendiguren-Galdospín, T. (2020). Fake news y coronavirus: detección de los principales actores y tendencias a través del análisis de las conversaciones en Twitter. El profesional de la información, 29(3), e290308. doi: https://doi.org/10.3145/epi.2020.may.08

Rebollo, M. Á. \& Vico, A. (2014). El apoyo social percibido como factor de la inclusión digital de las mujeres de entorno rural en las redes sociales virtuales. Comunicar, 22(43), 173-180. http://dx.doi.org/10.3916/C43-2014-17

Rodrich, R. (2012). Fundamentos de la Comunicación Institucional: una aproximación histórica y conceptual de la profesión, Revista de Comunicación, (11), 212-234. Recuperado de https://revistadecomunicacion.com/pdf/2012/Art212-234.pdf

Rodríguez Andrés, R. (2011). La efectividad del uso del miedo como factor persuasivo en la comunicación de riesgos en las crisis sanitarias. Revista de Comunicación y Salud, 1(2), 33-46. doi:10.35669/revistadecomunicacionysalud.2011.1(2).33-46

Rodríguez Andrés, R. (2017). ¿En camino hacia el gobierno 2.0?: análisis del uso de redes sociales por los ministerios españoles para fomentar la transparencia y la participación ciudadana. Estudios sobre el mensaje periodístico, 23(2), 937-954. doi: http://dx.doi.org/10.5209/ESMP.58025

Rodríguez González, M. del M., Marauri Castillo, I., Armentia Vizuet, J. I. \& Marín Murillo, F. (2020). De la crisis del pepino a la crisis del huevo. Aciertos y errores en la gestión de comunicación de crisis alimentarias. Revista de Comunicación y Salud, 10(1), 91-114. doi: http://doi.org/10.35669/rcys.2020.10(1).91-114

Rúas Araújo, X. \& Casero-Ripollés, A. (2018). Comunicación política en la época de las redes sociales: lo viejo y lo nuevo, y más allá. adComunica. Revista Científica de Estrategias, Tendencias e Innovación en Comunicación, (16), 21-24. doi: http://dx.doi.org/10.6035/2174-0992.2018.16.2 
Salaverría, R., Buslón, N., López-Pan, F., León, B., López-Goñi, I., \& Ervti, M. C. (2020). Desinformación en tiempos de pandemia: tipología de los bulos sobre la Covid-19. El profesional de la información, 29(3), e290315. doi: https://doi.org/10.3145/epi.2020.may.15

Stier, S., Bleier, A., Lietz, H., \& Strohmaier, M. (2018) Election Campaigning on Social Media: Politicians, Audiences, and the Mediation of Political Communication on Facebook and Twitter, Political Communication, 35(1), 50-74. doi: https://doi.org/10.1080/10584609.2017.1334728

Solito, L. \& Materassi, L. (2020). On a Municipality-Wide Scale. Social Media Use in Italian Municipalities: Information and Interaction. adComunica. Revista Científica de Estrategias, Tendencias $e$ Innovación en Comunicación, (20), 151-172. http://dx.doi.org/10.6035/2174-0992.2020.20.7.

Teddlie, C. \& Tashakkori, A. (2010). Overview of Contemporary Issues in Mixed Methods Research. En T. Abbas, \& C. Teddlie (Eds.), Handbook of Mixed Methods in Social and Behavioral Research (141), SAGE. doi: http://dx.doi.org/10.4135/9781506335193.n1

Torres Albero, C. (2017). Sociedad de la información y brecha digital en España. Panorama Social, 25, 1733.

Ure, M. (2016). La comunicación de la administración pública en las redes sociales: los casos de las ciudades de Buenos Aires y Bolonia. Palabra Clave, 19(1), 240-270. doi: 10.5294/pacla.2016.19.1.10

Vázquez Gestal, M., y Fernández Souto, A.B. (2014). El profesional de las Relaciones Públicas en Galicia: el caso de los principales ayuntamientos gallegos. Revista Internacional de Relaciones Públicas, 6(7), 25-38. doi: http://dx.doi.org/10.5783/RIRP-7-2014-03-25-38

Wimmer, R. D. \& Dominick, J. R. (1996). La investigación científica de los medios de comunicación: una introducción a sus métodos. Bosch.

World Health Organization (WHO). (2005). WHO outbreak communication guidelines. Geneva: WHO. Recuperado de https://www.who.int/csr/resources/publications/WHO CDS 2005 28/en/

Xifra, J. (2009). Comunicación proactiva. La gestión de conflictos potenciales en las organizaciones. Barcelona: Editorial Gedisa.

Xifra, J. (2020). Comunicación corporativa, relaciones públicas y gestión del riesgo reputacional en tiempos del Covid-19. El profesional de la información, 29(2), e290220. doi: https://doi.org/10.3145/epi.2020.mar.20

\section{Semblanza de los autores}

Andrea Castro-Martínez es Doctora en Publicidad y RRPP con Mención Internacional. Docente e investigadora en la Universidad de Málaga desde 2016. Anteriormente fue durante ocho años subdirectora de los grados de Comunicación (Periodismo y Publicidad y RRPP), Diseño Gráfico y Diseño de Interiores en EADE, sede de la Universidad de Gales en Málaga, donde también era docente de los grados y del MBA. Licenciada en Comunicación Audiovisual y en Publicidad y Relaciones Públicas por la Universidad de Málaga, ha cursado varios expertos en comunicación y marketing y un MBA. Ha trabajado en diferentes medios de comunicación y ha sido docente y consultora de marketing, publicidad y comunicación. Autora de más de una treintena de publicaciones, sus líneas de investigación se centran en relaciones públicas, 
comunicación estratégica, comunicación interna, nuevas formas publicitarias y comunicación con perspectiva de género.

Ignacio Morán-Urdiales es doctorando sobre comunicación política e institucional en la Universidad de Málaga. Director del Área de Comunicación de la Diputación de Málaga y jefe de prensa del Partido Popular de Málaga. Previamente, director de comunicación de la Mancomunidad de Municipios de la Costa del Sol Occidental y redactor en las agencias de noticias EFE y Europa Press. Licenciado en Periodismo. Experto Universitario en Historia Contemporánea y en Historia del Pensamiento.

Pablo Díaz-Morilla es Doctor en Publicidad y RRPP con Mención Internacional. Director y docente en los Grados en Comunicación (Periodismo y Publicidad y RRPP) en EADE University of Wales in Málaga desde 2007 y de las Enseñanzas Artísticas Superiores en Diseño en Institución docente malagueña León XIII desde 2014. Ha sido docente e investigador en el departamento de Comunicación Audiovisual y Publicidad de la Facultad de Ciencias de la Comunicación de la Universidad de Málaga. Autor de más de una quincena de publicaciones, sus líneas de investigación se centran en la aplicación de las nuevas formas publicitarias a la comunicación comercial, y en la comunicación organizacional y comunicación interna. 